\title{
Local properties of impurity and defects investigated by high pressure spectroscopy
}

\author{
Marek Grinberg \\ Institute of Experimental Physics, University of Gdańsk \\ Wita Stwosza 57, 80-952 Gdańsk, Poland \\ E-mail: fizmgr@univ.gda.pl
}

\begin{abstract}
Using the high-pressure spectroscopy, the pressure shifts of the luminescence related to the d-d transitions in transition metal ions and $d-f$ transitions in rare earth ones, which interact with the nearest neighbor host ions of dielectrics, are investigated. The results are obtained for $\mathrm{Ti}^{3+}, \mathrm{Ce}^{3+}, \mathrm{Pr}^{3+}$, and $\mathrm{Cr}^{3+}$ ions in various lattices.
\end{abstract}

Keywords: rare earth ion, transition metal ion, high-pressure spectroscopy.

Manuscript received 07.02.07; accepted for publication 27.09.07; published online 31.10.07.

Spectral properties of the dielectrics doped with transition elements are determined by the localized states related to unfilled internal electronic shell $3 \mathrm{~d}^{n}$ $(1 \leq n \leq 10)$ in the case of transition metal ions and $4 \mathrm{f}^{n}$ or $4 \mathrm{f}^{n-1} 5 \mathrm{~d}^{1} \quad(1 \leq n \leq 14)$ in the case of rare earth ions. Although the $3 \mathrm{~d}$ and $5 \mathrm{~d}$ electrons are localized at the transition ion, they extend enough to interact with the nearest neighbor host ions. As a result, the energetic structures of the transition metal and rare earth ion are different in different hosts. This sensitivity on the host lattice caused the development in crystal field theory. We have used the high hydrostatic pressure to change smoothly the crystal field and, in this manner, to control all environment-dependent interactions in the system. For applying pressure, we have used diamond anvil cell (DAC) that is the most useful equipment for high pressure optical measurements. Using our system, we can apply hydrostatic pressure up to $400 \mathrm{kbar}$. This paper summarize our results obtained for $\mathrm{Ti}^{3+}, \mathrm{Ce}^{3+}, \mathrm{Pr}^{3+}$, and $\mathrm{Cr}^{3+}$ ions in various lattices. The purpose of the present contribution is to show how high-pressure spectroscopy can be used to investigate the peculiarities of host materials.

We have investigated the pressure shift of the luminescence related to the $\mathrm{d}-\mathrm{d}$ transitions in the transition metal ions and $\mathrm{d}-\mathrm{f}$ transitions in the rare earth ions. The shifts were related to the pressure dependence of the cubic crystal field parameter $D q$. Since the crystal field strength is a power function of the central ion ligand distance, $R$, it is possible to relate the effect of pressure on the energetic structure of the transition ions to the elastic properties of a host material. One considers that pressure causes increasing the crystal field with the rate given by following relation:

$$
\frac{d D q}{d R}=-n D q \frac{1}{R} \frac{d R}{d p}=n D q \frac{K}{3 B_{0}}
$$

for transition metal ions and

$$
\frac{d \Delta E}{d p}=\left[n^{\prime} E_{c e n}+n E_{C R}\right] \frac{K}{3 B_{0}}=n\left[E_{C R}+\frac{n^{\prime}}{n} E_{c e n}\right] \frac{K}{3 B_{0}}
$$

for rare earth ions. Here, $B_{0}$ is bulk modulus of a host material. The dimensionless coefficient $K$ describes the way in which the macroscopic effect of pressure modifies the local environment of the central ion. $K=1, K<1$, and $K>1$ means that the compression of the ion - ligands system is the same, smaller, and larger than the compression of the bulk lattice.

Considering the pressure shifts of the characteristic emission bands of dopands, one can measure the local compression and the Grüneisen parameter. We have found that local compressibility of the coordination ion is about two times smaller than bulk compressibility of the material. The respective data obtained by us and taken from the literature are collected in Table. 
Table. Spectroscopic data and calculated parameters for transition metals and rare earth ions in different materials. Parameter $K$ is the relative compression of a coordination ion.

\begin{tabular}{|c|c|c|c|c|c|}
\hline Material & $\begin{array}{r}E_{\text {depr }}, \\
\left(\mathrm{cm}^{-1}\right)\end{array}$ & $\begin{array}{c}E_{e m} \\
\left(\mathrm{~cm}^{-1}\right)\end{array}$ & $\begin{array}{c}d E_{e m} / d p \\
\left(\mathrm{~cm}^{-1} / \mathrm{kbar}\right)\end{array}$ & $B_{0}$ (kbar) & $K$ \\
\hline YAG:Ce ${ }^{3+}$ & 27570 & $\begin{array}{l}19100 \\
17560\end{array}$ & $\begin{array}{l}-12.5 \\
-11.8\end{array}$ & 1870 & 0.51 \\
\hline GSAG: $\mathrm{Ce}^{3+}$ & 27000 & $\begin{array}{l}17340 \\
15250\end{array}$ & $\begin{array}{l}-8.3 \\
-7.5\end{array}$ & 1916 & 0.53 \\
\hline YAG: $\mathrm{Cr}^{3+}$ & & & & & 0.59 \\
\hline LLGG:Cr ${ }^{3+}$ & & 11700 & 13 & 1330 & $\begin{array}{l}0.69 \\
0.61\end{array}$ \\
\hline $\mathrm{Al}_{2} \mathrm{O}_{3}: \mathrm{Cr}^{3+}$ & & 13670 & 5.40 & 2420 & 0.68 \\
\hline $\mathrm{Al}_{2} \mathrm{O}_{3}: \mathrm{Ti}^{3+}$ & & 12450 & 5.93 & 2420 & 0.59 \\
\hline $\mathrm{YAlO}_{3}: \mathrm{Ti}^{3+}$ & & 16330 & 19.6 & 2004 & 1.1 \\
\hline $\mathrm{LiNbO}_{3}: \mathrm{Cr}^{3+}$ & & & 13.5 & 1058 & 0.57 \\
\hline $\mathrm{LiTaO}_{3}: \mathrm{Cr}^{3+}$ & 27125 & 10800 & 13.5 & 1057 & 0.54 \\
\hline YAG:Pr ${ }^{3+}$ & & 11020 & -14.1 & 1870 & 0.5 \\
\hline
\end{tabular}

The increase of stiffness of the impurity sites has been attributed to the increase of the energy of localized $3 \mathrm{~d}$ and $4 \mathrm{f}$ electrons caused by pressure-induced confinement of the system. Using the approximation where the local ion potential was presented by a quantum well, one performed model calculations, and an increase of local stiffness was obtained.

\section{Acknowledgements}

The research presented in this paper has been supported by the Polish State Committee for Scientific Research by grant No. 2P03 B057 23. 